\title{
Manganese (II) Chelate Functionalized Copper Sulfide Nanoparticles for Efficient Magnetic Resonance/ Photoacoustic Dual-Modal Imaging Guided Photothermal Therapy
}

\author{
Renfa Liu1, Lijia Jing1,3, Dong Peng ${ }^{2}$, Yong Li ${ }^{3}$, Jie Tian², Zhifei Dai ${ }^{1 凶}$ \\ 1. Department of Biomedical Engineering, College of Engineering, Peking University, Beijing 100871, China; \\ 2. Institute of Automation, Chinese Academy of Sciences, Beijing 100190, China; \\ 3. School of Life Science and Technology, Harbin Institute of Technology, Harbin 150080, China.
}

\begin{abstract}
$\bowtie$ Corresponding author: E-mail address: zhifei.dai@pku.edu.cn
(C) 2015 Ivyspring International Publisher. Reproduction is permitted for personal, noncommercial use, provided that the article is in whole, unmodified, and properly cited. See http://ivyspring.com/terms for terms and conditions.
\end{abstract}

Received: 2015.02.02; Accepted: 2015.06.05; Published: 2015.07.25

\begin{abstract}
The integration of diagnostic and therapeutic functionalities into one nanoplatform shows great promise in cancer therapy. In this research, manganese (II) chelate functionalized copper sulfide nanoparticles were successfully prepared using a facile hydrothermal method. The obtained ultrasmall nanoparticles exhibit excellent photothermal effect and photoaoustic activity. Besides, the high loading content of $\mathrm{Mn}$ (II) chelates makes the nanoparticles attractive $T_{1}$ contrast agent in magnetic resonance imaging (MRI). In vivo photoacoustic imaging (PAl) results showed that the nanoparticles could be efficiently accumulated in tumor site in $24 \mathrm{~h}$ after systematic administration, which was further validated by MRI tests. The subsequent photothermal therapy of cancer in vivo was achieved without inducing any observed side effects. Therefore, the copper sulfide nanoparticles functionalized with $\mathrm{Mn}(\mathrm{II})$ chelate hold great promise as a theranostic nanomedicine for MR/PA dual-modal imaging guided photothermal therapy of cancer.
\end{abstract}

Key words: Copper sulfide nanoparticles; Nanotheranostic agent; Photoacoustic imaging; Magnatic resonance imaging; Photothermal therapy.

\section{Introduction}

Photothermal therapy (PTT) employing near infrared (NIR) light-absorbing agents to generate heat and "boil" tumor cells has gained increasing attention for its minimal invasiveness and potential effectiveness [1, 2]. A large number of NIR-absorbing nanomaterials including organic nanomaterials and inorganic nanostructures have been explored for photothermal ablation of cancer [3-7]. In recent years, image-guided therapy has been developed as a new concept to achieve highly efficient and accurate treatments of cancers $[2,8]$. By integrating contrast enhanced medical imaging capability with therapeu- tic agent, the therapeutic efficiency can be greatly enhanced by monitoring the distribution of nanoparticles in real-time before, during and after the therapeutic process avoiding damage to surrounding normal tissues and cells $[9,10]$. Since no single imaging modality is perfect and sufficient enough to provide all aspects of anatomical and functional information, the development of multi-modal imaging guided therapy is clearly attractive [11-14].

As one of the most prominent imaging tools in daily clinics, magnetic resonance imaging (MRI) has become the preferred tool for detecting cancer owing 
to its excellent soft tissue contrast and unlimited penetration depth. However, the application of MRI is limited by its poor sensitivity [11,15]. Several groups have explored to combine optical imaging with MRI to compensate the innate limitations of MRI [16-18]. However, these traditional optical techniques have limited spatial resolution and penetration depth [19, 20]. Photoaoustic imaging (PAI) is an emerging technology that integrates both the merits of optical contrast and ultrasonic resolution [21, 22]. By providing high optical contrast images at a microscale resolution and a reasonable penetration depth, PAI is expected to be a promising candidate for combination with MRI [23-25].

Superparamagnetic iron oxide (SPIO) has been a prevailing MRI contrast agent since its development for liver imaging [26]. However, the inherent disadvantage of SPIO resulting from the negative contrast effect and magnetic susceptibility greatly limited its applications in MRI [27]. Gadolinium (III)-based chelates are another prevailing contrast agent for clinical MRI. Unfortunately, Gd(III) based agents are reported to be possibly involved in nephrogenic system fibrosis and thus restricted for clinical use by the FDA (Food and Drug Administration)[28]. As an essential element for human, manganese (II) possesses strong qualities for use in MRI comparable to Gd(III) [29], while the biocompatibility of $\mathrm{Mn}$ (II) is much better than that of $\mathrm{Gd}(\mathrm{III})$ [30]. Therefore, in recent years, increasing researches have been done to explore Mn(II) based agents for MRI [31-33].

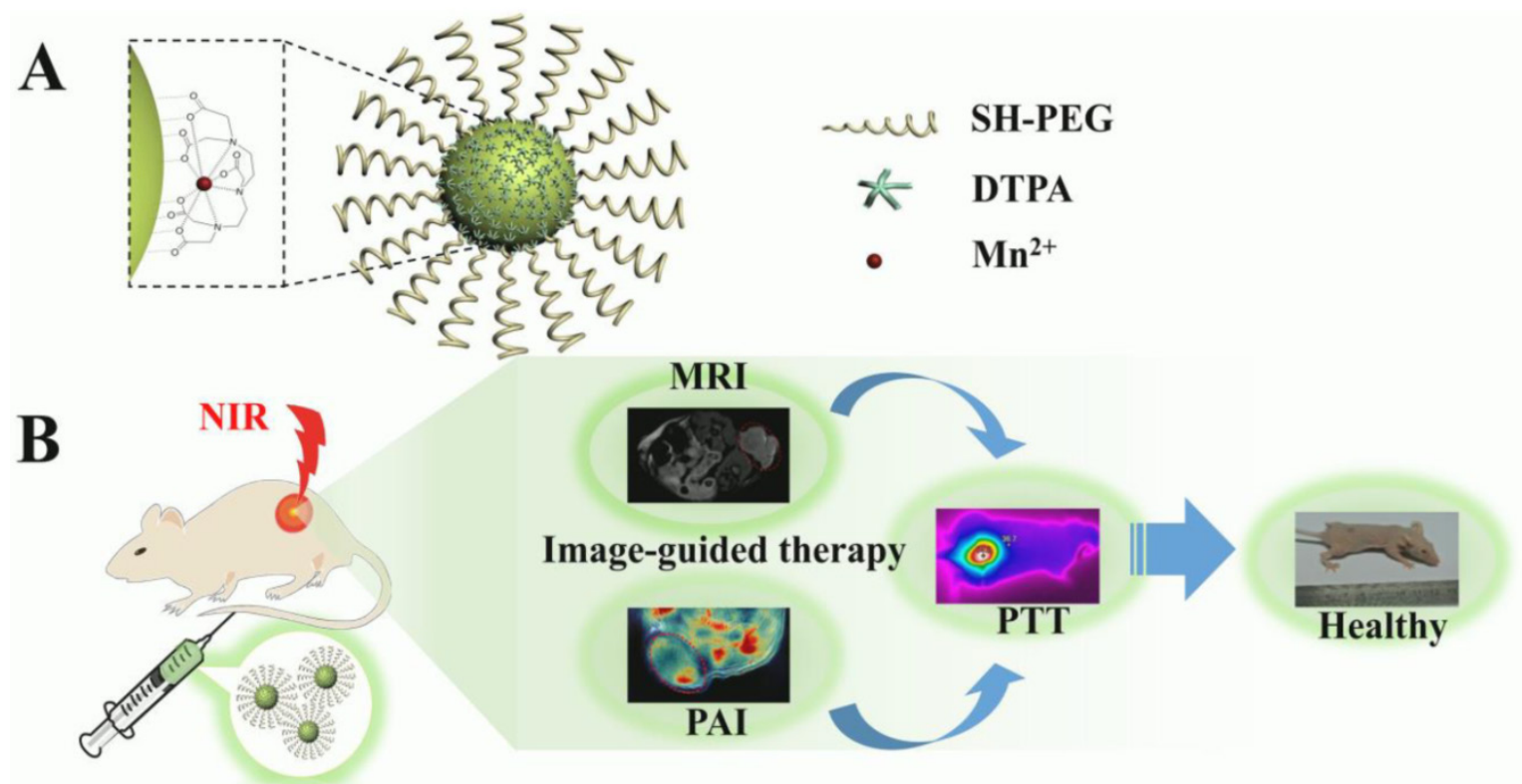

Copper sulfide nanoparticles (CuS NPs) are promising nanomaterials for PTT developed in recent years [34, 35]. Compared with the widely-explored gold nanostructures, CuS NPs are advantageous in regard to their ultrasmall size, low cost of production and unique optical properties without need for elaborate shape design [34]. CuS NPs-based nanoplatforms have been studied for image guided therapy, indicating their promising future in cancer theranostics [36]. Although several researches have been reported to utilize CuS NPs for PAI or MRI guided therapy [37-40], the application of CuS NPs for MR/PA dual-modal imaging guided cancer therapy through systematic administration has not yet been reported to the best of our knowledge.

Herein, a multifunctional nanoplatform based on CuS NPs functionalized with Mn(II) chelate and poly(ethylene glycol) (PEG) (CuS@MPG NPs) was developed for MR/PA dual-modal imaging guided PTT (Figure 1A\&B). The CuS@MPG NPs exhibit ultrasmall size, excellent NIR-absorbing capability and minimal cytotoxicity. The high loading content of Mn(II) chelates confers the CuS@MPG NPs relatively high relaxivity, making it promising non-Gd(III) MRI contrast agent. After i.v. injection into tumor-bearing mice, the CuS@MPG NPs could be efficiently accumulated in tumor, which was demonstrated by PAI and MRI results. The tumors were then irradiated with NIR laser and photothermally ablated without any observed side effects. Our results highlight the bright future of CuS@MPG NPs as a multifunctional nanoplatform for image-guided therapy. 


\section{Materials and Methods}

\section{Materials}

Copper (II) chloride $\left(\mathrm{CuCl}_{2}\right)$, sodium sulfide nonahydrate $\left(\mathrm{Na}_{2} \mathrm{~S} \cdot 9 \mathrm{H}_{2} \mathrm{O}\right)$, manganese acetate tetrahydrate $\left(\left(\mathrm{Mn}\left(\mathrm{CH}_{3} \mathrm{COO}\right)_{2} \cdot 4 \mathrm{H}_{2} \mathrm{O}\right)\right.$, diethylene triamine pentacetate acid (DTPA) was all obtained from Sinopharm Chemical reagent Co. LTD. Thiol-terminated methoxypoly(ethylene glycol) (mPEG-SH; molecular weight $5000 \mathrm{Da}$ ) was purchased from Beijing Kaizheng Biotech. Fluorescein poly(ethylene glycol) thiol (FITC-PEG-SH, molecular weight $5000 \mathrm{Da}$ ) was obtained from Shanghai ToYongBio. All chemicals are of analytical grade and used directly without further purification. Millipore quality deionized water (DI water) (resistivity is 18.2 $\mathrm{M} \Omega \cdot \mathrm{cm}$ ) was utilized in all experiments.

\section{Preparation of NPs}

$0.1 \mathrm{mmol}$ DTPA was dissolved in $84 \mathrm{~mL}$ DI water, and then $5 \mathrm{~mL}$ of $10 \mathrm{mM}$ manganese acetate solution were added. The solution were then stirred at 55 ${ }^{\circ} \mathrm{C}$ for $1 \mathrm{~h}$ to form stable Mn(II)-DTPA. $20 \mathrm{mg}$ mPEG-SH (for FITC-labeled CuS@MPG NPs, 5\% mPEG-SH was replaced with FITC-PEG-SH) and 10 $\mathrm{mL}$ of $10 \mathrm{mM} \mathrm{CuCl}_{2}$ solution were added and further stirred for $5 \mathrm{~min}$, followed by drop-wise addition of 1 $\mathrm{ml}$ of $0.1 \mathrm{M}$ of $\mathrm{Na}_{2} \mathrm{~S}$. After $5 \mathrm{~min}$, the reaction mixture was heated to $90{ }^{\circ} \mathrm{C}$ and stirred for $30 \mathrm{~min}$ until a dark-green solution was obtained. The mixture was then transferred to ice-cold water. Further dialysis was performed to exclude unreacted agents. The dialysis procedure was done against DI water with a relative sample to water volume ratio of 1: 200 for 48 hours. Water was refreshed regularly for at least eight times. After that, the samples were frozen in liquid nitrogen and lyophilized for $24 \mathrm{~h}$ using a SIM FD5-3 freeze drier. The powdery CuS@MPG NPs were stored at $4{ }^{\circ} \mathrm{C}$.

PEG-stabilized CuS NPs (PEG-CuS NPs) was synthesized using previously described method [35]. For Mn(II)-DTPA, 0.1 mmol DTPA was dissolved in $10 \mathrm{~mL}$ phosphate based solution (PBS), after which 0.1 mmol manganese acetate was added. The solution were then stirred at $55{ }^{\circ} \mathrm{C}$ for $2 \mathrm{~h}$ to form stable Mn(II)-DTPA. The Mn(II)-DTPA solution was stored at $4{ }^{\circ} \mathrm{C}$ before using.

\section{Characterizations of CuS@MPG NPs}

Transmission electron microscopy (TEM) and high resolution TEM (HRTEM) images of CuS@MPG NPs were obtained using a Hitachi H9000 TEM (Japan) operated at $300 \mathrm{kV}$. The hydrodynamic diameter of the CuS@MPG NPs was determined with a PALS/90Plus dynamic light scattering (DLS) analyzer
(Brookhaven Instruments Co., USA). The absorption spectra of CuS@MPG NPs were recorded using an UV-vis-NIR spectrometer (Evolution 220, Thermo Scientific) using quartz cuvettes with an optical path of $1 \mathrm{~cm}$. The concentration of $\mathrm{Cu}$ and $\mathrm{Mn}$ element was determined by inductively coupled plasma atomic emission spectroscopy (ICP-AES) (PROFILE SPEC, Leeman).

\section{Photothermal Heating Experiments in Vitro}

The CuS@MPG NPs and PEG-CuS NPs of various concentrations were added in quartz cuvettes $(2.0 \mathrm{~mL})$, irradiated by continuous-wave diode NIR laser with a center wavelength of $808 \pm 10 \mathrm{~nm}$ and output power of $2 \mathrm{~W}$ for $5 \mathrm{~min}$. The temperature was measured by a thermocouple inserted into the solution perpendicular to the light path every $10 \mathrm{~s}$.

\section{Cellular experiments}

The photothermal ablation effect of CuS@MPG NPs to cancer cells was evaluated with the MDA-MB-231 cells. The cell line was derived from human breast cancer cells. MDA-MB-231 cells were seeded onto a 24-well plate at a density of $5 \times 10^{4}$ cells/well, a day before the experiment. After incubated with CuS@MPG NPs or PEG-CuS NPs, cells were then exposed to NIR laser $\left(808 \mathrm{~nm}, 0.64 \mathrm{~W} / \mathrm{cm}^{2}\right)$ for $10 \mathrm{~min}$ and stained with calcein AM (calcein acetoxymethyl ester) and PI (propidium iodide) to verify the photohyperthermic effect on cancer cells.

To determine the cellular uptake of CuS@MPG NPs, MDA-MB-231 cells pre-seeded in 12-well plates were incubated with CuS@MPG NPs for $4 \mathrm{~h}$. After washing cells with PBS for 3 times, cells were stained with 40, 6-diamidino-2-phenylindole (DAPI) and then imaged by a confocal laser scanning microscope (Nikon, A1R-si).

The photothermal cytotoxicity of CuS@MPG NPs to MDA-MB-231 cells under NIR irradiation was further quantitatively investigated using the conventional 3-(4, 5-dimethylthiazol-2-yl)-2, 5-diphenyltetrazolium bromide (MTT) assay. MDA-MB-231 cells were dispensed into a 96-well flat bottom plate $(1 \times$ $10^{4}$ cells/well), 1 day before the experiment. The cells were then exposed to CuS@MPG NPs or PEG-CuS NPs of various concentrations and irradiated by NIR laser $\left(0.64 \mathrm{~W} / \mathrm{cm}^{2}\right)$ for $10 \mathrm{~min}$ and the cell viabilities were determined by the standard MTT assay according to the manufacturer suggested procedures.

\section{PAI Procedure}

All phantom and in vivo mouse imaging experiments were performed using a real-time multispectral optoacoustic tomographic (MSOT) imaging system (inVision 128, iThera Medical GmbH, Neuherberg, Germany).The phantom is made of polyure- 
thane, specially designed to mimic the shape, size and optical properties of the mouse, containing two inner cylindrical channels for holding the control medium (PBS) and the contrast agent dissolved in PBS.

Female Balb/c nude mice were purchased from Beijing Vital River Laboratories and all animal experiments were conducted under protocols approved by Peking University Laboratory Animal Center. Xenograft mice models were established by injecting subcutaneously 5-6 × 106 MDA-MB-231 cells into the right flank of mouse. For in vivo PAI tests, MDA-MB-231 tumor-bearing mice were i.v. injected with CuS@MPG NPs (0.48mg/mouse). A volume ROI consisting of transverse slices with a step size of 0.3 $\mathrm{mm}$ spanning through the tumor region was selected by manual inspection of live MSOT images. Multispectral imaging was performed before injection, and $1 \mathrm{~h}, 3 \mathrm{~h}, 5 \mathrm{~h}, 8 \mathrm{~h}$ and $24 \mathrm{~h}$ after injection. Images were reconstructed using a model-based approach [41, 42].

\section{MRI Procedure}

Both in vitro and in vivo MRI tests were performed on a 7.0-T MRI system designed for small animal imaging (Bruker). $\mathrm{T}_{1}$-weighted images of CuS@MPG NPs at various concentrations in pure water were acquired using the following parameters: repetition time $(\mathrm{TR})=1500 \mathrm{~ms}$, echo time $(\mathrm{TE})=7 \mathrm{~ms}$. Relaxivity $\mathrm{r}_{1}$ was calculated from the slopes of relaxation time $\left(\mathrm{T}_{1}\right)$ versus the concentration of $\mathrm{Mn}(\mathrm{II})$. For in vivo MRI tests, MDA-MB-231 tumor-bearing mice were treated with an i.v. injection of CuS@MPG NPs (0.48 mg/mouse) and $\mathrm{T}_{1}$-weighted images were acquired before and $24 \mathrm{~h}$ after injection.

\section{Pharmacokinetic and Biodistribution Study}

Wistar rats were obtained from Beijing Vital River Laboratories. To determine the circulation time, CuS@MPG NPs $(0.48 \mathrm{mg} / \mathrm{mouse})$ were injected into tumor free rats via the tail vein. Blood samples $(0.3 \mathrm{~mL})$ were collected at the time points of $0.2,0.5,1,2,4,8$, 12 , and $24 \mathrm{~h}$ after intravenous injection. The pharmacokinetics profile of CuS@MPG NPs in bloodstream was finally evaluated by measuring the $\mathrm{Cu}$ content in blood over the course of $24 \mathrm{~h}$ via ICP method after decomposing the blood samples by aqua regia.

For biodistribution assay, MDA-MB-231 tumor-bearing mice were sacrificed at $24 \mathrm{~h}$ after i.v. injection of CuS@MPG NPs $(0.48 \mathrm{mg} /$ mouse, $\mathrm{n}=3)$. Major organs including heart, liver, spleen, lung, kidney, and tumor were collected, weighed, and then solubilized by aqua regia. The concentrations of $\mathrm{Cu}$ and $\mathrm{Mn}$ in those tissue lysate samples were measured by ICP. The levels of CuS@MPG NPs in blood and various organs are presented as the percentage of injected dose per gram of tissue (\%ID /g).

\section{PTT in Vivo}

MDA-MB-231 tumor-bearing mice were i.v. injected with CuS@MPG NPs solution dispersed in PBS $(0.48 \mathrm{mg} / \mathrm{mouse})$. In control groups, mice were injected with the same volume of PBS. Mice with and without CuS@MPG NPs injection were irradiated with the $808 \mathrm{~nm}$ NIR laser at a power density of 0.64 $\mathrm{W} / \mathrm{cm}^{2}$ for $10 \mathrm{~min}$. Temperature elevation of tumor area was monitored in a real-time way with a thermometer inserted into the tumor and an infrared thermal camera (Fluke Ti27). The tumor sizes were measured by a caliper every other day and calculated as the tumor volume $=$ (tumor length) $\times$ (tumor width $)^{2} / 2$. Mice with tumor sizes exceeding 1000 $\mathrm{mm}^{3}$ were euthanatized according to the standard animal protocol. Relative tumor volumes were calculated as $\mathrm{V} / \mathrm{V}_{0}\left(\mathrm{~V}_{0}\right.$ was the tumor volume when the treatment was initiated). Vital organs including heart, liver, spleen, lung and kidney of mice in agent+laser group and untreated healthy mice were collected and stained using standard hematoxylin \& eosin (H\&E) staining protocol.

\section{Results and discussion}

\section{Preparation and Characterization of CuS@MPG NPs}

The CuS@MPG NPs were prepared using a previously reported method with some modification [38]. In brief, the chelating agent of diethylene triamine pentaacetic acid (DTPA) was mixed with manganous acetate to form stable Mn(II)-DTPA, followed by addition of mPEG-SH and copper chloride. Finally, a deep-green CuS@MPG NPs solution was formed upon addition of sodium sulfide. The excess agents were removed through dialyzing against pure water for $48 \mathrm{~h}$. The obtained NPs aqueous solution was then freeze-dried to powder and stored at $4{ }^{\circ} \mathrm{C}$. The TEM images revealed that the CuS@MPG NPs displayed a rather uniform size distribution with an average size at $\sim 9 \mathrm{~nm}$ (Figure 2A). The effective diameter of CuS@MPG NPs in water measured with DLS test was $31.8 \mathrm{~nm}$ (Figure S1), larger than TEM diameter. The difference between these two tests was probably due to the PEGylated modification on the surface of CuS@MPG NPs, which was invisible under TEM. HRTEM images of CuS@MPG NPs showed that the lattice spacing of (102) plane was approximately 0.30 $\mathrm{nm}$ (Figure 2B), in agreement with previously reported lattice spacing of (102) plane of hexagonal CuS nanostructures $(0.305 \mathrm{~nm})[43]$. The powdery CuS@MPG NPs were quite stable and could be stored for several months without any obvious change (data not shown). The CuS@MPG NPs could be well re-dispersed in various solutions and exhibit a quite 
similar NIR absorption characteristics with a strong absorption peak at $\sim 930 \mathrm{~nm}$ (Figure 2C\&D), consistent with previously reported CuS NPs [35]. Besides, the size and size distribution of CuS@MPG NPs suspended in these solutions were relatively stable without any obvious changes (Figure S1), indicating the colloidal stability of CuS@MPG NPs.

Using an ICP-AES, the molar ratio of copper to manganese in CuS@MPG NPs was determined to be 5.15. Assuming the density of CuS NPs to be 4.6 $\mathrm{g} / \mathrm{cm}^{3}$ [34], each CuS@MPG NP was estimated to contain $2140 \mathrm{Mn}$ (II) ions combined with the average diameter of $9 \mathrm{~nm}$. The high loading content of $\mathrm{Mn}$ (II) chelates is expected to confer CuS@MPG NPs ability to enhance the contrast in MRI images. We determined the longitudinal relaxivity of CuS@MPG NPs at various concentrations under a 7.0-T MRI scanner (Figure 3A\&B). The relaxivity $\mathrm{r}_{1}$ was measured to be $7.10 \mathrm{mM}^{-1} \mathrm{~s}^{-1}$, which is a relatively high value among existing Mn(II)-based contrast agents [29]. The excellent ability to shorten longitudinal relaxation times of protons from bulk water makes the CuS@MPG NP promising $\mathrm{Mn}(\mathrm{II})$-based contrast agents for $\mathrm{T}_{1}$-weighted MRI.
A

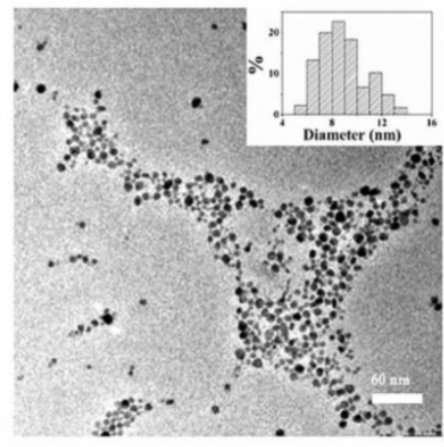

C

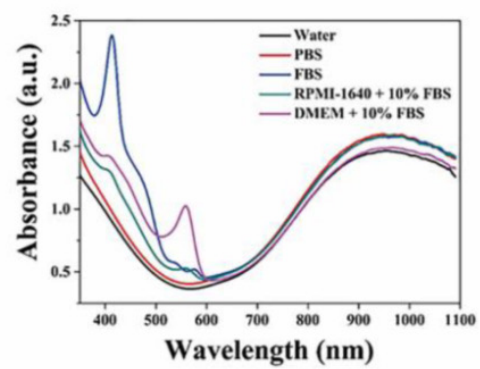

B

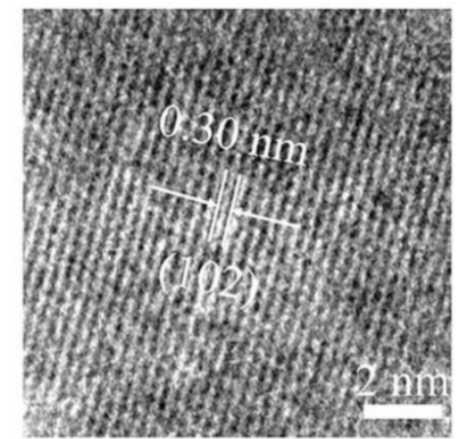

D

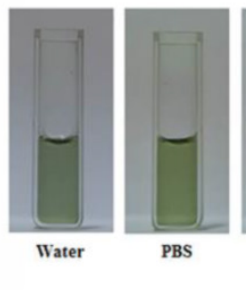

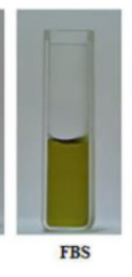
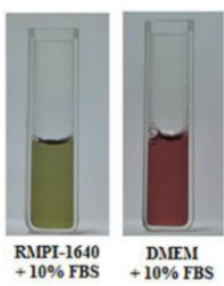

Figure 2. Characterization of CuS@MPG NPs: (A) TEM images and particle size distribution based on TEM results (Inset); (B) HRTEM images; (C) UV-vis-NIR spectra; (D) Photos of CuS@MPG NPs dispersed in water, PBS, fetal bovine serum (FBS), RMPI-1640 culture media containing 10\% FBS and DMEM culture media containing 10\% FBS.

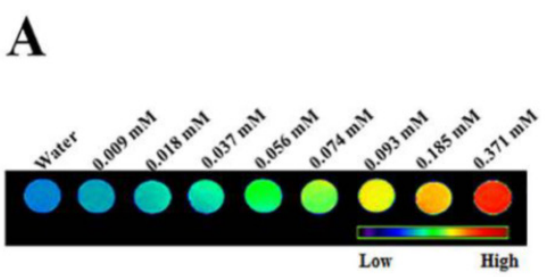

C

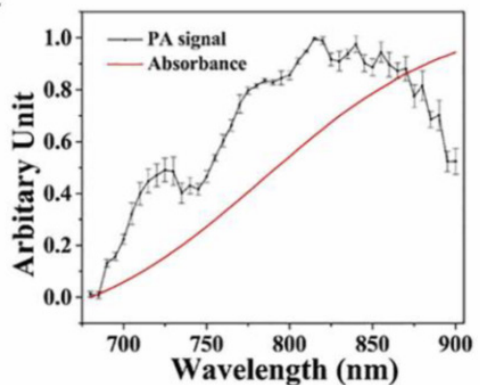

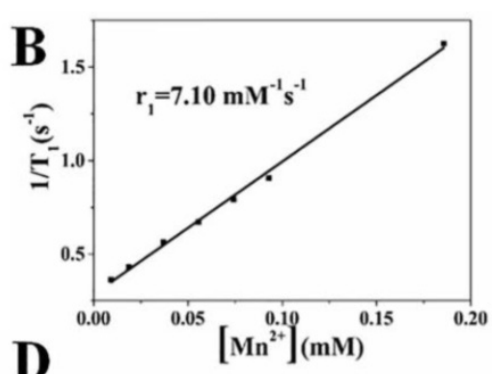

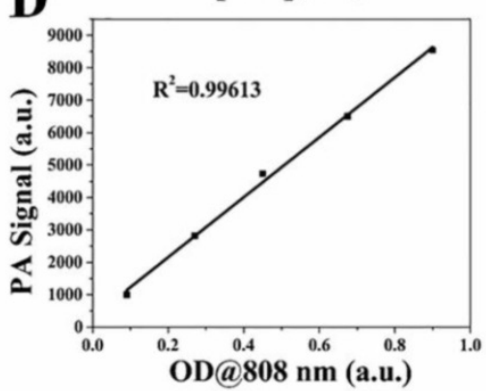

Figure 3. (A) In vitro $\mathrm{T}_{1}$-weighted MRI images of CuS@MPG NPs aqueous solutions with increasing concentrations of Mn(II). (B) Linear relationship between $\mathrm{T}_{\text {I }}$ relaxation rate $\left(1 / T_{1}\right)$ and Mn(II) concentrations in CuS@MPG NPs aqueous solutions. (C) Normalized PA signal and absorbance of CuS@MPG NPs solutions as a function of wavelength. (D) PA signal intensity as a function of optical densities (OD) at $808 \mathrm{~nm}$. 
The CuS NPs were reported previously as an excellent PAI contrast agent [37, 39, 40]. In order to demonstrate the PA activity of CuS@MPG NPs, a phantom measurement was performed, in the 680-900 $\mathrm{nm}$ wavelength range, at five different concentrations using literature-described protocols [44]. After image reconstruction, it was shown that the PA activity of CuS@MPG NPs was wavelength-dependent (Figure 3C). The PA spectrum exhibited a strong correlation with optical absorption spectrum. The small difference in trend between NIR absorbance and PA intensities was possibly due to light fluence changes resulting from variations in laser intensity [44]. Besides, the PA signals were linearly correlated with concentrations of NPs (Figure 3D). These phantom results showed that the CuS@MPG NPs possessed good PA contrast enhanced capability.

\section{NIR Photothermal Heating Effects}

One of the most striking feature of CuS@MPG NPs is their phothermal conversion ability, which can be used for PTT of cancer. To investigate temperature elevation induced by CuS@MPG NPs in combination with NIR laser irradiation, the aqueous solution of CuS@MPG NPs with different concentrations (with same total volume of $2.0 \mathrm{~mL}$ ) in quartz cuvettes was exposed to a continuous-wave fiber-coupled diode laser $(808 \mathrm{~nm}, 2 \mathrm{~W})$ for $5 \mathrm{~min}$. Figure $4 \mathrm{~A}$ showed that temperature collected by a digital thermometer every $10 \mathrm{~s}$ of samples under irradiation rose with increasing
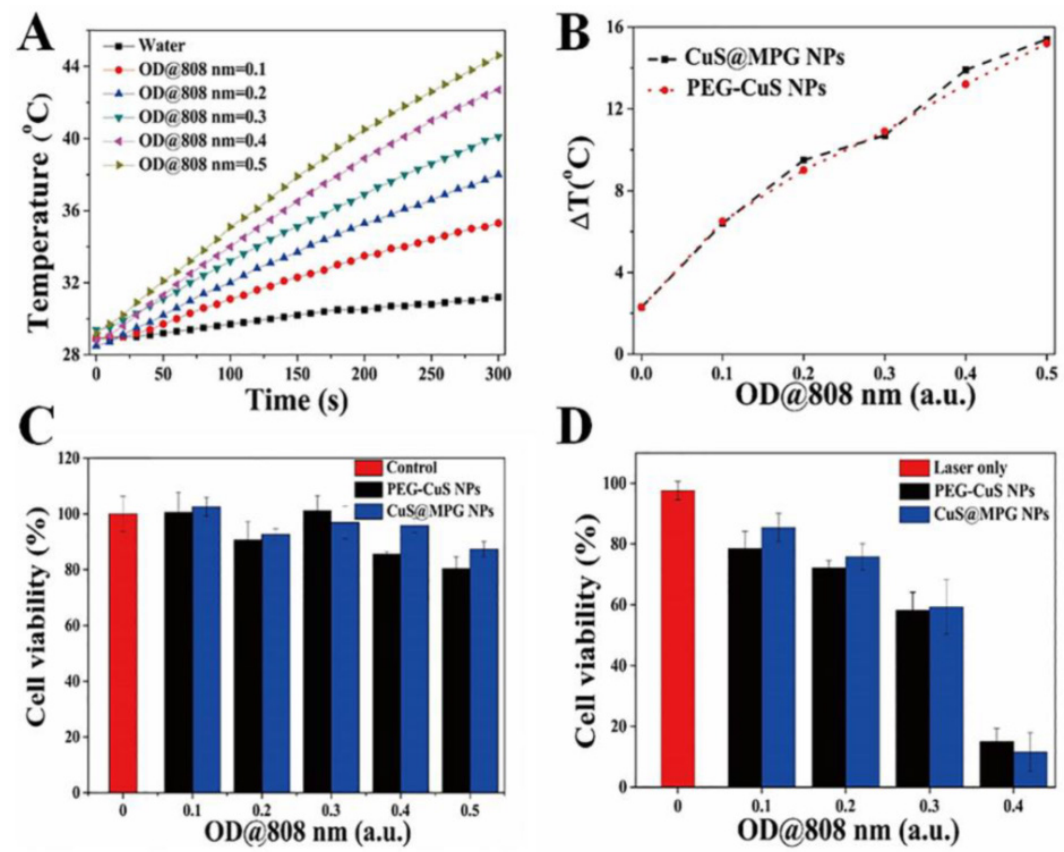

Figure 4. (A) The temperature of the aqueous dispersion of CuS@MPG NPs with different concentrations changed as time. (B) Plot of temperature elevation $(\Delta T)$ of CuS@MPG NPs and PEG-CuS NPs over a period of 5 min versus OD at $808 \mathrm{~nm}$. (C) In vitro cytotoxicity of CuS@MPG NPs and PEG-CuS NPs to MDA-MB-231 cells. (D) Photothermal cytotoxicity to MDA-MB-231 cells as a function of laser irradiation time and NPs concentration. time. The temperature elevation of $6.4,9.5,10.7,13.9$ and $15.4{ }^{\circ} \mathrm{C}$ was achieved under the NIR laser irradiation of CuS@MPG NPs aqueous solution for $5 \mathrm{~min}$ with OD at $808 \mathrm{~nm}$ of $0.1,0.2,0.3,0.4,0.5$, respectively (Figure 4B). On the contrary, the temperature changed only $2.3^{\circ} \mathrm{C}$ when pure water was exposed to the laser, verifying the excellent photothermal activity of CuS@MPG NPs. The temperature elevation of CuS@MPG NPs is very close to that of PEG-CuS NPs with the same OD at $808 \mathrm{~nm}$ (Figure 4B).

The photothermal ablation ability of CuS@MPG NPs was then evaluated at cellular level with MDA-MB-231 cells. CuS@MPG NPs could be efficiently internalized by MDA-MB-231 cells after incubation for $4 \mathrm{~h}$ (Figure S2), and then heat could be generated with CuS@MPG NPs upon laser irradiation to induce localized cellular death. As shown in Figure S3, cells treated with NPs plus NIR irradiation experienced substantial cellular death while no apparent cellular death was observed in cells treated with NPs or laser alone.

The standard MTT assay was then conducted to quantitatively determine the relative viabilities of MDA-MB-231 cells under various treatments. The concentration of Mn in CuS@MPG NPs solution at the $\mathrm{OD}_{808 \mathrm{~nm}}$ of 0.5 was measured to be $\sim 0.058 \mathrm{mM}$. As shown in Figure 4C \& Figure S4, cell viabilities of MDA-MB-231 cells remained higher than $80 \%$ when treated with CuS@MPG NPs, bulk PEG-CuS NPs and $\mathrm{Mn}(\mathrm{II})$-DTPA at comparable concentrations, indicating negligible cytotoxicity of CuS@MPG NPs. However, less than $20 \%$ of MDA-MB-231 cells survived when treated with CuS@MPG NPs at the $\mathrm{OD}_{808 \mathrm{~nm}}$ of 0.4 under NIR irradiation $\left(808 \mathrm{~nm}, 0.64 \mathrm{~W} \mathrm{~cm}^{-2}\right)$ for $10 \mathrm{~min}$ (Figure 4D). Besides, the photothermal cytotoxicity of CuS@MPG NPs against MDA-MB-231 cells was dose-dependent similar to PEG-CuS NPs. These results demonstrated the potential of CuS@MPG NPs as a photothermal agent for localized ablation of cancer cells under NIR laser irradiation.

\section{Dual-modal Imaging in Vivo}

The ideal nano-agent for cancer therapy should be able to be efficiently accumulated in tumor site after systematic administration. Utilizing the real-time MSOT imaging system, the in vivo behavior of CuS@MPG NPs was investigated. PA images were collected before and after a single i.v. injection of 
CuS@MPG NPs. As shown in Figure 5A, the initial PA signal in the tumor site before injection was rather weak. But just $1 \mathrm{~h}$ after injection, the signal clearly showed up and gradually increased over time (Figure 5A\&B). While the overall PA signal in the tumor region nearly reaches the highest in $5 \mathrm{~h}$, the signals expanded throughout the region in the following time, suggesting that large amounts of CuS@MPG NPs circulated in the blood and then accumulated at the tumor site. It provided a good evidence of the high efficiency of tumor targeting of these nanoparticles. CuS@MPG NPs could be passively accumulated into tumor due to the enhanced permeation and retention (EPR) effect.

The MSOT results were further confirmed by in vivo MRI results. Utilizing the relatively high $\mathrm{r}_{1}$ relaxivity of CuS@MPG NPs, we performed in vivo MRI tests under the 7.0-T MR imaging system. As shown in Figure $5 \mathbf{C} \& \mathbf{D}$, compared to images collected pre-injection, significantly enhanced $\mathrm{T}_{1}$-weighted $\mathrm{MR}$ signals were observed in the tumor $24 \mathrm{~h}$ post-injection, which was correlated with MSOT results. It suggested that the CuS@MPG NPs remained in the tumor sites as long as $24 \mathrm{~h}$ to provide adequate time for the following treatment. All these results demonstrated the potential of CuS@MPG NPs as MR/PA dual-modal imaging contrast agents.

\section{In Vivo Blood Circulation and Biodistribution}

The in vivo behavior of CuS@MPG NPs was also investigated using standard ICP method. As shown in Figure S5A, CuS@MPG NPs displayed a prolonged

blood circulation half-time of $3.56 \pm 0.43 \mathrm{~h}$. The biodistribution data of CuS@MPG NPs in vivo at $24 \mathrm{~h}$ post-injection indicated that the highest uptake of CuS@MPG NPs was found in reticuloendothelial systems (RES) such as liver and spleen, followed by tumor (Figure S5B). The higher uptake of CuS@MPG NPs by tumor can be attributed to the EPR effect and the longer circulation time, in agreement with the in vivo imaging results. Besides, no significant difference between the biodistribution of $\mathrm{Cu}$ and $\mathrm{Mn}$ content was observed, indicating the relatively well stability of CuS@MPG NPs. Therefore, the passively targeted CuS@MPG NPs can be used to display the tumor area by MR/PA dual-modal imaging to guide the NIR laser irradiation for photothermal ablation of tumors without damaging surrounding healthy tissues.

\section{PTT in Vivo}

Both of the PAI and MRI results indicated that CuS@MPG NPs can be efficiently accumulated in the tumor site $24 \mathrm{~h}$ after i.v. injection, which may be the optimal time for further photothermal ablation of tumor. We then performed in vivo PPT on MDA-MB-231 tumor-bearing nude mouse xenograft models. The tumor-bearing mice were divided into four groups: control group, laser-only group, agent-only group and agent+laser group. Mice in agent-only group and agent+laser group were i.v. injected with CuS@MPG NPs (200 $\mu \mathrm{L}$, $0.48 \mathrm{mg} /$ mouse), while $200 \mu \mathrm{L}$ PBS per mouse in control group and laser-only group. After $24 \mathrm{~h}$, tumors in laser-only group and agent+laser group were exposed to the NIR laser $(808 \mathrm{~nm}$,
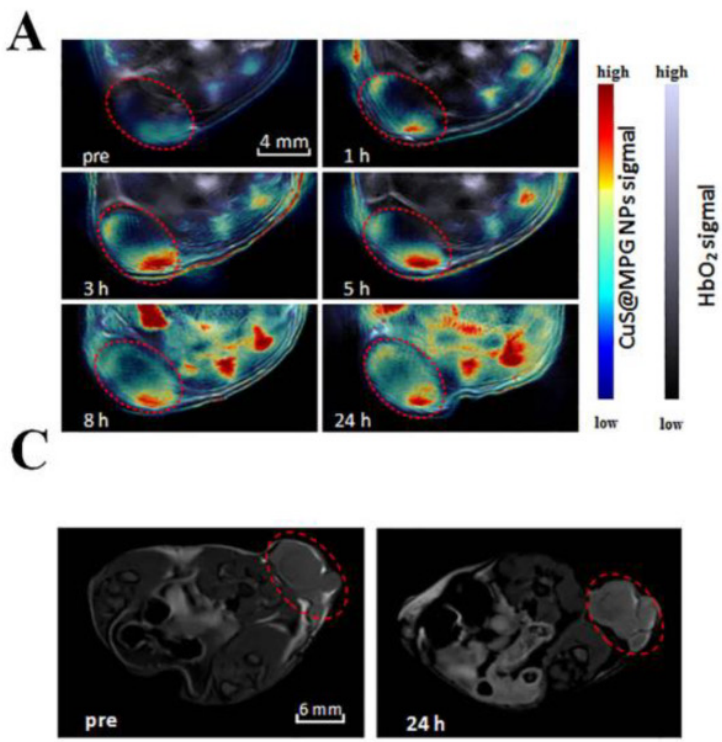
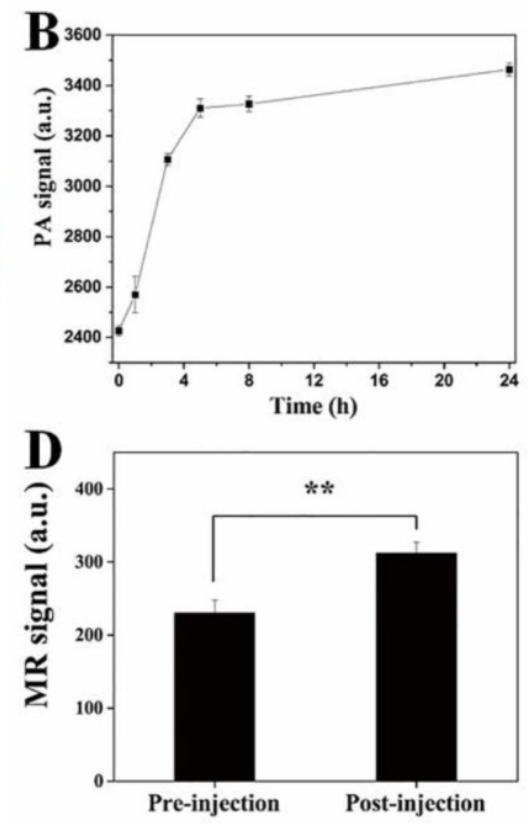

Figure 5. (A) PA images collected by MOST imaging system before (pre) and at various time points ( $1 \mathrm{~h}, 3 \mathrm{~h}, 5 \mathrm{~h}, 8 \mathrm{~h}, 24 \mathrm{~h})$ after i.v. injection of CuS@MPG NPs. (B) PA signals in the tumor region as a function of time post-injection. (C) $\mathrm{T}_{1}$-weighted MRI images before (pre) and $24 \mathrm{~h}$ after i.v. injection of CuS@MPG NPs. (D) MR signals in the tumor region pre-injection and $24 \mathrm{~h}$ post-injection $(P<0.05$, Student's t-test). $\left.0.64 \mathrm{~W} / \mathrm{cm}^{2}, 10 \mathrm{~min}\right)$. The temperature in tumor region was recorded in real-time with a thermometer inserted into tumor and an infrared thermal camera (Figure 6A\&B). The center tumor temperature in agent+laser group reached $\sim 54.5^{\circ} \mathrm{C}$ in $10 \mathrm{~min}$ and remained above $50{ }^{\circ} \mathrm{C}$ for more than $6 \mathrm{~min}$, obviously beyond the threshold cancer cells could tolerate. On the contrary, tumor temperature in laser-only group did not exceed $41^{\circ} \mathrm{C}$.

The high tumor temperature in agent+ laser group resulted in tumor growth inhibition 
in the following days after treatment. As shown in Figure 6C, mice tumors in agent+laser group were obviously inhibited after treatment and completely eliminated within the first week. In contrast, tumor growth in laser-only group and agent-only group was not affected compared with control group. This indicated only CuS@MPG NPs injection plus NIR irradiation were able to account for tumor inhibition, while either of them alone could not.

During our experimental process, no body weight loss or other abnormalities were observed in the four groups (Figure 6D\&E), suggesting all treatments during PTT were acceptable to tumor-bearing mice. The histological examination further demonstrated biocompatibility of our treatments (Figure 6F). No noticeable abnormality including inflammation, cell necrosis and apoptosis were observed in heart, liver, spleen, lung and kidney.

These results all demonstrated the potential of CuS@MPG NPs as a theranostic agent for image-guided photothermal therapy. After systematic administration, CuS@MPG NPs firstly act as a MR/PA dual-modal contrast agent. Utilizing the synergistic advantage of MRI and PAI, the in vivo behavior of CuS@MPG NPs is monitored and the location and size of tumor are identified. After that, CuS@MPG NPs can then be applied for PTT under guidance of MR/PA dual-modal imaging results and real-time monitoring of infrared thermal imaging, resulting in greatly improved therapeutic efficiency of tumor without damaging surrounding normal tissues.
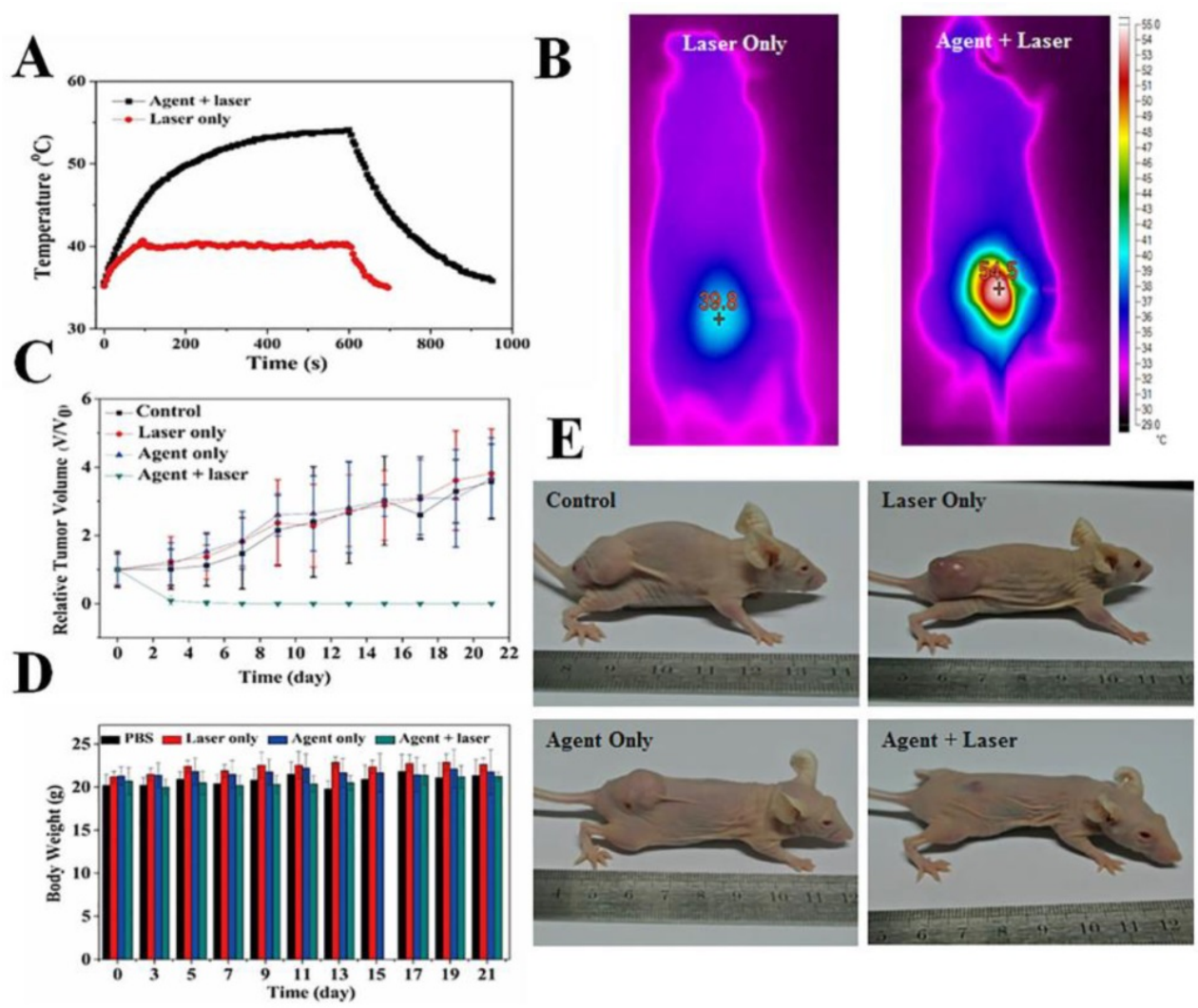

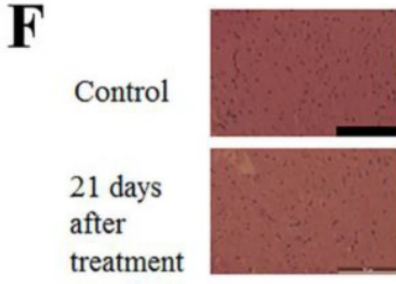

Heart

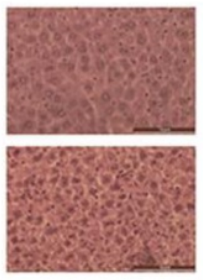

Liver

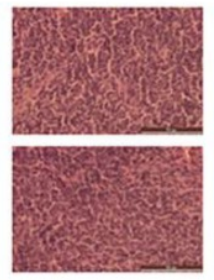

Spleen

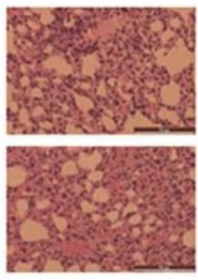

Lung

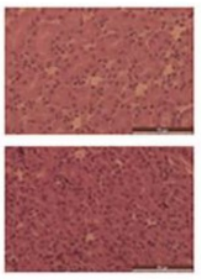

Kidney

Figure 6. In vivo photothermal therapy using CuS@MPG NPs. (A) Temperature evolution on tumors of MDA-MB-231 tumor-bearing mice with and without CuS@MPG NPs monitored with a thermometer during laser treatment. (B) The infrared thermal images from tumor region in laser-only group and agent+laser group, respectively ( $808 \mathrm{~nm}$ laser, $0.64 \mathrm{~W} / \mathrm{cm}^{2}$ for $10 \mathrm{~min}$. The thermal images were taken in the last second of laser irradiation). (C) The tumor growth curves and (D) body weights of mice bearing MDA-MB-231 tumors after different treatments indicated $(n=5)$. (E) Representative photographs of mice in four groups 21 days after treatments. (F) Histological examination of vital organs (heart, liver, spleen, lung and kidney) of mice in agent+laser group at 21 th day after treatments. Untreated healthy mouse was used as control. Scale bar $=50 \mu \mathrm{m}$. 


\section{Conclusion}

In summary, we have successfully prepared $\mathrm{Mn}$ (II) chelate functionalized copper sulfide nanoparticles for combined MR/PA dual-modal imaging-guided phototherapy. The resultant CuS@MPG NPs exhibit ultrasmall size, high photothermal conversion efficiency and minimal in vitro cytotoxicity. The in vivo MRI and PAI results indicated that the nanoparticles could be accumulated in the tumor region by EPR effect in $24 \mathrm{~h}$ after i.v. injection, which was further verified by ICP results. The in vivo PTT results showed that the tumors were efficiently ablated. No recurrences or side effects were observed during the experiments. Hence, our results promise the exploration of $\mathrm{CuS}$ NPs-based systems as theranostic nanomedicine for accurate and personalized treatment of cancer.

\section{Supplementary Material}

Supplementary Figures S1-S5.

http://www.thno.org/v05p1144s1.pdf

\section{Acknowledgement}

This work was financially supported by National Natural Science Foundation for Distinguished Young Scholars (Grant No. 81225011), State Key Program of National Natural Science of China (Grant No. 81230036), National Natural Science Foundation of China (Grant No. 21273014) and the Foundation for Innovative Research Groups of the National Natural Science Foundation of China (Grant No. 81421004).

\section{Competing Interests}

The authors have declared that no competing interest exists.

\section{References}

1. Nolsoe CP, Torppedersen S, Burcharth F, Horn T, Pedersen S, Christensen $\mathrm{NEH}$, et al. Interstitial Hyperthermia of Colorectal Liver Metastases with a Us-Guided Nd-Yag Laser with a Diffuser Tip - a Pilot Clinical-Study. Radiology 1993; 187: 333-7.

2. Ke H, Wang J, Dai Z, Jin Y, Qu E, Xing Z, et al. Gold-nanoshelled microcapsules: a theranostic agent for ultrasound contrast imaging and photothermal therapy. Angew Chem Int Ed Engl. 2011; 50: 3017-21.

3. Markovic ZM, Harhaji-Trajkovic LM, Todorovic-Markovic BM, Kepić DP, Arsikin KM, Jovanović SP, et al. In vitro comparison of the photothermal anticancer activity of graphene nanoparticles and carbon nanotubes. Biomaterials. 2011; 32: 1121-9.

4. Gong H, Dong Z, Liu Y, Yin S, Cheng L, Xi W, et al. Engineering of Multifunctional Nano-Micelles for Combined Photothermal and Photodynamic Therapy Under the Guidance of Multimodal Imaging. Advanced Functional Materials. 2014

5. Zha Z, Deng Z, Li Y, Li C, Wang J, Wang S, et al. Biocompatible polypyrrole nanoparticles as a novel organic photoacoustic contrast agent for deep tissue imaging. Nanoscale. 2013; 5: 4462-7.

6. Ma Y, Liang X, Tong S, Bao G, Ren Q, Dai Z. Gold Nanoshell Nanomicelles for Potential Magnetic Resonance Imaging, Light-Triggered Drug Release, and Photothermal Therapy. Advanced Functional Materials. 2013; 23: 815-22.

7. Yi DK, Sun I-C, Ryu JH, Koo H, Park CW, Youn I-C, et al. Matrix metalloproteinase sensitive gold nanorod for simultaneous bioimaging and photothermal therapy of cancer. Bioconjugate chemistry. 2010; 21: 2173-7.
8. Sun $\mathrm{Y}, \mathrm{Cao} \mathrm{W}, \mathrm{Li} \mathrm{S}$, Jin $\mathrm{S}, \mathrm{Hu} \mathrm{K}, \mathrm{Hu} \mathrm{L}$, et al. Ultrabright and multicolorful fluorescence of amphiphilic polyethyleneimine polymer dots for efficiently combined imaging and therapy. Sci Rep. 2013; 3: 3036.

9. Chen Z, Ma L, Liu Y, Chen C. Applications of functionalized fullerenes in tumor theranostics. Theranostics. 2012; 2: 238.

10. Guo CX, Jin YS, Dai ZF. Multifunctional Ultrasound Contrast Agents for maging Guided Photothermal Therapy. Bioconjugate Chem. 2014; 25: 840-54.

11. Jennings LE, Long NJ. 'Two is better than one' - probes for dual-modality molecular imaging. Chemical Communications. 2009: 3511-24.

12. Tang $Y$, Zhang $C$, Wang J, Lin $X$, Zhang $L$, Yang $Y$, et al. MRI/SPECT/Fluorescent Tri-Modal Probe for Evaluating the Homing and Therapeutic Efficacy of Transplanted Mesenchymal Stem Cells in a Rat ischemic Stroke Model. Advanced Functional Materials. 2014

13. Xiao OF, Zheng XP, Bu WB, Ge WQ Zhang SJ, Chen F, et al. A Core/Satellite Multifunctional Nanotheranostic for in Vivo Imaging and Tumor Eradication by Radiation/Photothermal Synergistic Therapy. Journal of the American Chemical Society. 2013; 135: 13041-8.

14. Ke HT, Wang JR, Tong S, Jin YS, Wang SM, Ou EZ, et al. Gold Nanoshelled Liquid Perfluorocarbon Magnetic Nanocapsules: a Nanotheranostic Platform for Bimodal Ultrasound/Magnetic Resonance Imaging Guided Photothermal Tumor Ablation. Theranostics. 2014; 4: 12-23.

15. Judenhofer MS, Wehrl HF, Newport DF, Catana C, Siegel SB, Becker M, et al. Simultaneous PET-MRI: a new approach for functional and morphological imaging. Nat Med. 2008; 14: 459-65.

16. Koffie RM, Farrar CT, Saidi LJ, William CM, Hyman BT, Spires-Jones TL. Nanoparticles enhance brain delivery of blood-brain barrier-impermeable probes for in vivo optical and magnetic resonance imaging. P Natl Acad Sci USA. 2011; 108: 18837-42.

17. Chen Y-J, Wu S-C, Chen C-Y, Tzou S-C, Cheng T-L, Huang Y-F, et al. Peptide-based MRI contrast agent and near-infrared fluorescent probe for intratumoral legumain detection. Biomaterials. 2014; 35: 304-15.

18. Yamane T, Hanaoka K, Muramatsu Y, Tamura K, Adachi Y, Miyashita Y, et al. Method for Enhancing Cell Penetration of Gd3+-based MRI Contrast Agents by Conjugation with Hydrophobic Fluorescent Dyes. Bioconjugate Chemistry. 2011; 22: 2227-36.

19. Mallidi S, Luke GP, Emelianov S. Photoacoustic imaging in cancer detection, diagnosis, and treatment guidance. Trends Biotechnol. 2011; 29: 213-21.

20. Ke H, Xing Z, Zhao B, Wang J, Liu J, Guo C, et al Quantum-dot-modified microbubbles with bi-mode imaging capabilities. Nanotechnology. 2009; 20: 425105 .

21. Yang J-M, Favazza C, Chen R, Yao J, Cai X, Maslov K, et al. Simultaneous functional photoacoustic and ultrasonic endoscopy of internal organs in vivo. Nature medicine. 2012; 18: 1297-302.

22. Nie L, Chen X. Structural and functional photoacoustic molecular tomography aided by emerging contrast agents. Chem Soc Rev. 2014; 43: 7132-70.

23. Kircher MF, de la Zerda A, Jokerst JV, Zavaleta CL, Kempen PJ, Mittra E, et al. A brain tumor molecular imaging strategy using a new triple-modality MRI-photoacoustic-Raman nanoparticle. Nat Med. 2012; 18: 829-U235.

24. Qin H, Zhou T, Yang SH, Chen Q, Xing D. Gadolinium(III)-gold nanorods for MRI and photoacoustic imaging dual-modality detection of macrophages in atherosclerotic inflammation. Nanomedicine-Uk. 2013; 8: 1611-24.

25. Cheng L, Gong H, Zhu WW, Liu JJ, Wang XY, Liu G, et al. PEGylated Prussian blue nanocubes as a theranostic agent for simultaneous cancer imaging and photothermal therapy. Biomaterials. 2014; 35: 9844-52.

26. Na HB, Song IC, Hyeon T. Inorganic Nanoparticles for MRI Contrast Agents. Adv Mater. 2009; 21: 2133-48.

27. Na HB, Lee JH, An KJ, Park YI, Park M, Lee IS, et al. Development of a T-1 contrast agent for magnetic resonance imaging using $\mathrm{MnO}$ nanoparticles. Angew Chem Int Edit. 2007; 46: 5397-401.

28. Sieber MA, Steger-Hartmann T, Lengsfeld P, Pietsch H. Gadolinium-based contrast agents and NSF: evidence from animal experience. Journal of magnetic resonance imaging : JMRI. 2009; 30: 1268-76.

29. Kueny-Stotz M, Garofalo A, Felder-Flesch D. Manganese-Enhanced MRI Contrast Agents: From Small Chelates to Nanosized Hybrids. Eur J Inorg Chem. 2012;: 1987-2005.

30. Tan M, Ye Z, Jeong EK, Wu X, Parker DL, Lu ZR. Synthesis and evaluation of nanoglobular macrocyclic $\mathrm{Mn}$ (II) chelate conjugates as non-gadolinium(III) MRI contrast agents. Bioconjug Chem. 2011; 22: 931-7.

31. Chen $Y$, Yin $\mathrm{Q}$ Ji X, Zhang $\mathrm{S}$, Chen $\mathrm{H}$, Zheng $\mathrm{Y}$, et al. Manganese oxide-based multifunctionalized mesoporous silica nanoparticles for $\mathrm{pH}$-responsive MRI, ultrasonography and circumvention of MDR in cancer cells. Biomaterials. 2012; 33: 7126-37.

32. Sharma VK, Gokyar S, Kelestemur Y, Erdem T, Unal E, Demir HV. Manganese doped fluorescent paramagnetic nanocrystals for dual-modal imaging. Small. 2014; 10: 4961-6.

33. Jing LJ, Liang XL, Li XD, Lin L, Yang YB, Yue XL, et al. Mn-porphyrin Conjugated $\mathrm{Au}$ Nanoshells Encapsulating Doxorubicin for Potential Magnetic Resonance Imaging and Light Triggered Synergistic Therapy of Cancer. Theranostics. 2014; 4: 858-71.

34. Li Y, Lu W, Huang Q, Li C, Chen W. Copper sulfide nanoparticles for photothermal ablation of tumor cells. Nanomedicine-Uk. 2010; 5: 1161-71.

35. Zhou M, Zhang R, Huang M, Lu W, Song S, Melancon MP, et al. A chelator-free multifunctional [64Cu]CuS nanoparticle platform for simultaneous micro-PET/CT imaging and photothermal ablation therapy. J Am Chem Soc. 2010; 132: 15351-8. 
36. Goel S, Chen F, Cai W. Synthesis and biomedical applications of copper sulfide nanoparticles: from sensors to theranostics. Small. 2014; 10: 631-45.

37. Ku G, Zhou M, Song S, Huang Q, Hazle J, Li C. Copper sulfide nanoparticles as a new class of photoacoustic contrast agent for deep tissue imaging at 1064 nm. Acs Nano. 2012; 6: 7489-96

38. Zhang S, Zha Z, Yue X, Liang X, Dai Z. Gadolinium-chelate functionalized copper sulphide as a nanotheranostic agent for MR imaging and photothermal destruction of cancer cells. Chem Commun (Camb). 2013; 49: 6776-8.

39. Zha Z, Zhang S, Deng Z, Li Y, Li C, Dai Z. Enzyme-responsive copper sulphide nanoparticles for combined photoacoustic imaging, tumor-selective chemotherapy and photothermal therapy. Chem Commun (Camb). 2013; 49: 3455-7.

40. Yang K, Zhu L, Nie L, Sun X, Cheng L, Wu C, et al. Visualization of protease activity in vivo using an activatable photo-acoustic imaging probe based on CuS nanoparticles. Theranostics. 2014; 4: 134.

41. Rosenthal A, Razansky D, Ntziachristos V. Fast Semi-Analytical Model-Based Acoustic Inversion for Quantitative Optoacoustic Tomography. Ieee T Med Imaging. 2010; 29: 1275-85.

42. Buehler A, Rosenthal A, Jetzfellner T, Dima A, Razansky D, Ntziachristos V. Model-based optoacoustic inversions with incomplete projection data. Med Phys. 2011; 38: 1694-704.

43. Zhu H, Ji X, Yang D, Ji Y, Zhang H. Novel CuS hollow spheres fabricated by a novel hydrothermal method. Microporous and mesoporous materials. 2005; 80: 153-6.

44. Ho CJH, Balasundaram G, Driessen W, McLaren R, Wong CL, Dinish US, et al. Multifunctional Photosensitizer-Based Contrast Agents for Photoacoustic Imaging. Sci Rep-Uk. 2014; 4. 